\title{
A Novel Artificial Bee Colony Algorithm for Function Optimization
}

\author{
Song Zhang and Sanyang Liu \\ School of Mathematics and Statistics, Xidian University, Xian 710071, China \\ Correspondence should be addressed to Song Zhang; yxszhang@163.com \\ Received 6 October 2014; Revised 1 March 2015; Accepted 11 March 2015 \\ Academic Editor: Sishaj P. Simon
}

Copyright (C) 2015 S. Zhang and S. Liu. This is an open access article distributed under the Creative Commons Attribution License, which permits unrestricted use, distribution, and reproduction in any medium, provided the original work is properly cited.

\begin{abstract}
It is known that both exploration and exploitation are important in the search equations of ABC algorithms. How to well balance the two abilities in the search process is still a challenging problem in ABC algorithms. In this paper, we propose a novel artificial bee algorithm named as "NABC," by incorporating the information of the global best solution into the solution search equation of the onlookers stage to improve the exploitation. At the same time, we improve the search equation of the employed bees to keep the exploration. The experimental results of NABC tested on a set of 11 numerical benchmark functions show good performance and fast convergence in solving function optimization problems, compared with variant ABC, DE, and PSO algorithms. The application of NABC on solving five standard knapsack problems shows its effectiveness and practicability.
\end{abstract}

\section{Introduction}

Intelligent algorithms, such as genetic algorithm (GA) [1], particle swarm optimization (PSO) [2], ant colony optimization (ACO) [3], and Biogeography-Based Optimization (BBO) [4], have shown great success in solving optimization problems which are nonconvex, discontinuous, nondifferentiable, and so on. At the same time, they have many advantages, such as simplicity, ease of implementation, and outstanding performance. Inspired by the intelligent behaviors of honey bee swarms, Karaboga in 2005 [5] proposed the artificial bee colony $(\mathrm{ABC})$ algorithm for function optimizations. A set of experimental results [6-9] show that $A B C$ algorithm is competitive to some other intelligent algorithms. So the $\mathrm{ABC}$ algorithm has captured many attentions and has been applied to solve neural networks, flowshop scheduling problems, constrained optimization problems [10-12], and so on.

We know that both exploration and exploitation are necessary for an intelligent algorithm. In order to achieve good performances on problem optimizations in practice, the two abilities should be well balanced, while the two abilities contradict each other and the solution search equations of $A B C$ algorithm are good at exploration but poor at exploitation. So, many researchers mended the search solutions to improve the exploitation and the convergence during the past decades. Inspired by PSO, Zhu and Kwong [13] took advantage of the information of the global best solution to improve the performance of the $\mathrm{ABC}$ algorithm named GABC. Basturk and Karaboga [14] proposed a modified ABC algorithm by controlling the frequency of perturbation and introducing the ratio of the variance operator. Gao et al. proposed a novel $A B C$ method called EABC [15] to further improve the performance of $A B C$. In $E A B C$, in order to balance the exploration and the exploitation, they presented two new search equations to generate candidate solutions in the employed bee phase and the onlookers phase, respectively. Karaboga and Gorkemli presented a new version of $\mathrm{ABC}$ algorithm named quick artificial bee colony (qABC) [16], which modeled the behavior of onlooker bees more accurately and improved the performance of $\mathrm{ABC}$ in terms of local search ability. And the performance of the $\mathrm{qABC}$ depended on the neighborhood radius.

In this paper, we propose two new solution search equations in the stage of the employed bees and the onlookers, respectively, in order to improve the exploitation and keep the exploration. We name the novel ABC algorithm as "NABC." The experiment results tested on 11 numerical function optimizations show that the novel $\mathrm{ABC}$ algorithm is superior to the standard $\mathrm{ABC}$ algorithm and other improved $\mathrm{ABC}$ 
algorithms in most cases. And it is better than variant DE and PSO algorithms. At the same time, five knapsack problems are solved by $\mathrm{ABC}$ and $\mathrm{NABC}$, which shows that NABC is superior to $\mathrm{ABC}$ on solving engineering optimization problems.

The rest of this paper is organized as follows. Section 2 summarizes the standard ABC algorithm. The novel ABC algorithm named NABC is presented and analyzed in Section 3. Section 4 presents and discusses the experimental results of 11 benchmark functions. Section 5 shows the application of NABC on knapsack problems. Finally, the conclusion is drawn in Section 6.

\section{Standard ABC Algorithm}

Initialization is the first step in the standard ABC algorithm [5]. The position of a food source represents a possible solution to the optimization problem. Each food source is exploited by only one employed bee, so the number of employed bees named $\mathrm{SN}$ is equal to the number of food sources. And a random initial population of $\mathrm{SN}$ food sources is generated as follows:

$$
x_{i, j}=x_{\min , j}+\operatorname{rand}(0,1) *\left(x_{\max , j}-x_{\min , j}\right),
$$

where $i \in(1,2, \ldots, \mathrm{SN}), j \in(1,2, \ldots, D)$, and $D$ is the dimension of the numerical function; $x_{\max , j}$ and $x_{\min , j}$ are the lower and upper bounds of $x_{i, j}$.

Second is the search processes of the employed bees, where the onlookers and the scouts are started. The standard $\mathrm{ABC}$ algorithm uses the following equation to produce a candidate food source position from the old one:

$$
V_{i, j}=x_{i, j}+\phi_{i, j} *\left(x_{i, j}-x_{k, j}\right),
$$

where $k \in(1,2, \ldots, \mathrm{SN})$ and $j \in(1,2, \ldots, D)$ are random indexes; $k$ is different from $i ; \phi_{i, j}$ is a random number in the range $[-1,1]$. After each candidate source position is produced and evaluated by the employed bees, its performance is compared with that of the old food source position. If the new food source position has an equal or better quality than the old source position, the old one is replaced by the new one. Otherwise, the old one is retained. The greedy selection is used.

Third, the employed bees transfer their food information to the onlookers. The onlookers tend to further search the food around the selected food source. The onlooker chooses a food source depending on the probability value associated with that food source:

$$
p_{i}=\frac{\text { fitness }_{i}}{\sum_{i=1}^{n} \text { fitness }_{i}},
$$

where fitness $s_{i}$ is the fitness value of the solution which is obtained by the following equation:

$$
\text { fitness }_{i}= \begin{cases}\frac{1}{1+f_{i}}, & f_{i} \geq 0 \\ 1+\left|f_{i}\right|, & f_{i}<0\end{cases}
$$

where $f_{i}$ is the objective function value. If the new food source position has an equal or better quality than the old source position, the old one is replaced by the new one. Otherwise, the old one is retained which is the same as the employed bees stage.

At last, if a position cannot be improved further through a number of cycles, which is called Limit for abandonment, that food source is abandoned accordingly. The scout will discover a new food source position randomly using (1) to replace it.

\section{A Novel Artificial Bee Colony Algorithm (NABC)}

3.1. Modified Search Solutions. The exploration is the ability of investigating the various unknown regions in the solution space. So it is also very important in ABC algorithm. To retain the exploration, we present a modified search equation of the employed bees as follows:

$$
V_{i, j}=x_{r, j}+\phi_{i, j} *\left(x_{r, j}-x_{k, j}\right),
$$

where the index $r, k$ is randomly chosen from $(1,2, \ldots, \mathrm{SN})$, and different from the index $i . j \in(1,2, \ldots, D)$ is a randomly chosen index. $\phi_{i, j}$ is a random number in the range $[-1,1]$.

Differential evolution (DE) [17] is a simple and efficient evolutionary algorithm for many optimization problems in the real-world applications [18-22]. Inspired by it, many researchers improved variant intelligent algorithms. So inspired by DE and various modified search equations of the $\mathrm{ABC}$ algorithms and taking advantage of the information of the global best solution to guide the search of the candidate solutions, a new equation is proposed to serve as the search equation of onlookers, as follows:

$$
V_{i, j}=x_{\text {best }, j}+\phi_{i, j} *\left(x_{\text {best }, j}-x_{k, j}\right),
$$

where the index $k$ is a mutually exclusive integer randomly chosen from $(1,2, \ldots, \mathrm{SN})$, and different from the index $i$. $x_{\text {best }, j}$ is the $j$ th element of the global best solution and $j \in(1,2, \ldots, D)$ is a randomly chosen index. $\phi_{i, j}$ is a random number in the range $[-1,1]$. We name the novel ABC algorithm using (5) to replace (2) on the stage of the employed bees and using (6) to replace (2) on the stage of the onlookers as NABC, which can drive the new candidate solution only around the best solution of the previous iteration. Therefore, the modified solution search equation described by (6) can increase the exploitation of $\mathrm{ABC}$ algorithm greatly. The best solution in the current population is a very useful source which can be used to improve the convergence performance. Therefore, we modify the solution search equation by applying the global best solution to guide the search of new candidate solutions in order to improve the exploitation. At the same time, the exploration is improved by (5) from the experiments in Section 4.

The new candidate solution is generated around $x_{i, j}$ in GABC [13]. It is different from (6) in which the one is

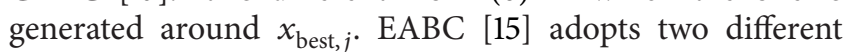
search equations based on different the emphases in the different search stages. NABC uses (5) and (6) to generate the new solution in the stages of employed bees and onlookers, respectively. In the $\mathrm{qABC}[16], x_{N_{m}}^{\text {best }}$ represents the best 
TABLE 1: Benchmark functions used in experiments.

\begin{tabular}{lccc}
\hline Fun & Name & Search range & Min \\
\hline$f_{1}=\sum_{i=1}^{n} x_{i}^{2}$ & Sphere & {$[-100,100]$} & 0 \\
$f_{2}=\sum_{i=1}^{n} i x_{i}^{2}$ & Sumsquare & {$[-10,10]$} & 0 \\
$f_{3}=\sum_{i=1}^{n}\left|x_{i}\right|+\prod_{i=1}^{n}\left|x_{i}\right|$ & Schwefel2.22 & {$[-10,10]$} & 0 \\
$f_{4}=\max _{\{}\left\{\left|x_{i}\right|, 1 \leq i \leq n\right\}$ & Schwefel2.21 & {$[-100,100]$} & 0 \\
$f_{5}=\sum_{i=1}^{n}\left(\left\lfloor x_{i}+0.5\right]\right)^{2}$ & Step & {$[-100,100]$} & 0 \\
$f_{6}=\sum_{i=1}^{n}\left[100\left(x_{i}^{2}-x_{i+1}\right)^{2}+\left(x_{i}-1\right)^{2}\right]$ & Rosenbrock & {$[-5,10]$} & 0 \\
$f_{7}=\sum_{i=1}^{n}\left[x_{i}^{2}-10 \cos \left(2 \pi x_{i}\right)+10\right]$ & Rastrigin & {$[-5.12,5.12]$} & 0 \\
$f_{8}=\frac{1}{4000} \sum_{i=1}^{n} x_{i}^{2}-\prod_{i=1}^{n} \cos \left(\frac{x_{i}}{\sqrt{i}}\right)+1$ & Griewank & {$[-600,600]$} & 0 \\
$f_{9}=418.98288727243369 * n-\sum_{i=1}^{n} x_{i} \sin \left(\sqrt{\left|x_{i}\right|}\right)$ & Schwefel2.26 & {$[-500,500]$} & 0 \\
$f_{10}=-20 \exp \left(-0.2 \sqrt{\frac{1}{n} \sum_{i=1}^{n} x_{i}^{2}}\right)-\exp \left(\frac{1}{n} \sum_{i=1}^{n} \cos \left(2 \pi x_{i}\right)\right)+20+e$ & Ackley & {$[-32,32]$} & 0 \\
$f_{11}=\frac{1}{n} \sum_{i=1}^{n}\left(x_{i}^{4}-16 x_{i}^{2}+5 x_{i}\right)$ & Himmelblau & {$[-5,5]$} & -78.33236 \\
\hline
\end{tabular}

solution among the neighbors of $x_{m}$ and itself $\left(N_{m}\right)$. But in the NABC, $x_{\text {best, } j}$ represents the global best solution. At the same time, in the stages of employed bees and onlookers, the new search equations used in $\mathrm{qABC}$ are different from these of the NABC adopted.

3.2. The Novel ABC Algorithm (NABC). The complete computational procedure of the novel $\mathrm{ABC}$ algorithm (NABC) is outlined as follows.

Algorithm 1 (NABC). Consider the following steps.

Step 1 (initialization). Preset SN, Limit, and Maxcycle.

Step 2. Randomly generate $\mathrm{SN}$ solutions from the search space to create an initial population $P$.

Step 3. Calculate the function values $f$ of the initial population $P$.

Step 4. The employed bees stage. Generate a new solution $v_{i, j}$ by (5), if $f\left(v_{i, j}\right)<f\left(x_{i, j}\right), x_{i, j}=v_{i, j}$, $^{\text {rail }}{ }_{i}=1$, else trail ${ }_{i}=$ trail $_{i}+1$.

Step 5. Calculate the probability values $p_{i}$ for the solutions $x_{i, j}$ by (3).

Step 6 (the onlookers stage). If rand $<p_{i}$, generate a new solution $v_{i, j}$ by (6), if $f\left(v_{i, j}\right)<f\left(x_{i, j}\right), x_{i, j}=v_{i, j}$, trail $_{i}=1$, else $\operatorname{trail}_{i}=\operatorname{trail}_{i}+1$.

Step 7 (the scouts stage). If trail > Limit, replace $x_{i, j}$ with a new produced solution by (1).
Step 8. If cycle number $>$ Maxcycle, stop and memorize the best solution achieved so far; otherwise, go to Step 2 .

\section{Numerical Functions and Experimental Results}

4.1. Numerical Functions. It is applied to minimize a set of 11 scalable benchmark functions in Table 1 to test the efficiency of the novel $\mathrm{ABC}$ algorithm. The smaller the final result, the better it is.

4.2. Parameter Settings. A set of experiments tested on 11 numerical benchmark functions are performed in this section to compare the performance of the novel $\mathrm{ABC}$ algorithm with that of other improved $\mathrm{ABC}$ algorithms. Functions $f_{1}-f_{4}$ are unimodal. Function $f_{5}$ is a discontinuous step function. The Rosenbrock function of $f_{6}$ which is unimodal when $D=$ 2 and 3 may have multiple minima in the high dimension case [18]. Functions $f_{7}-f_{11}$ are multimodal. To compare with other variance algorithms, the dimension $D$ of every function is 30,60 , or 100 , respectively, the same as [15]. The dimension $D$ of $f_{11}$ is 150,300 , or 500 . The maximum number of generations is 1500,3000 , or 5000 , respectively. The population size is 100 , namely, $\mathrm{SN}=50$, and Limit is 200 , which is the same as [15]. Each of the experiments is repeated 30 times independently.

4.3. Experimental Results. Figure 1 graphically presents the convergence curves of $f_{1}-f_{10}$ functions, respectively. We can see from Figure 1 that, in the later stage of evolution, the standard ABC algorithm enters a long period of stagnation. 

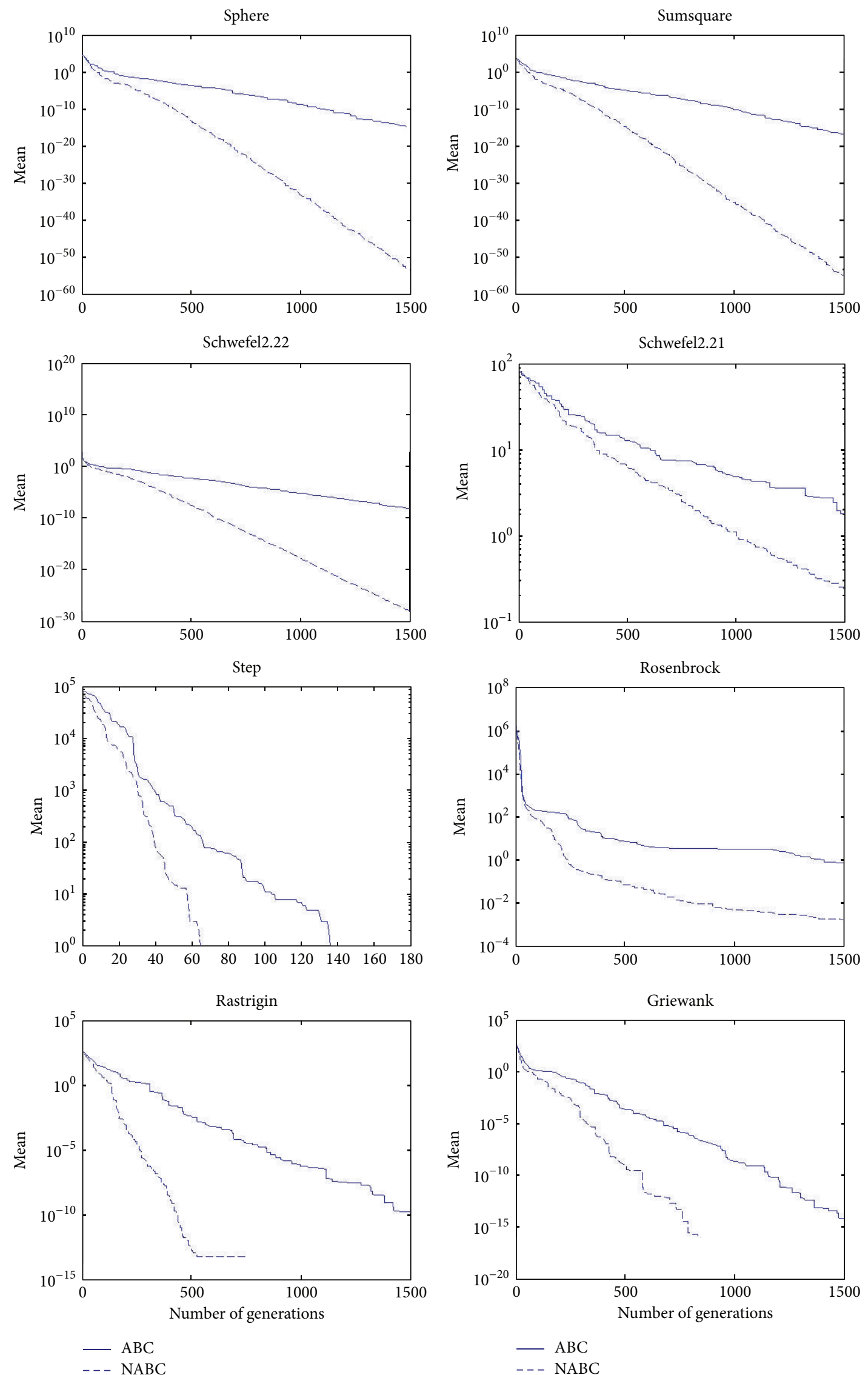

FIGURe 1: Continued. 

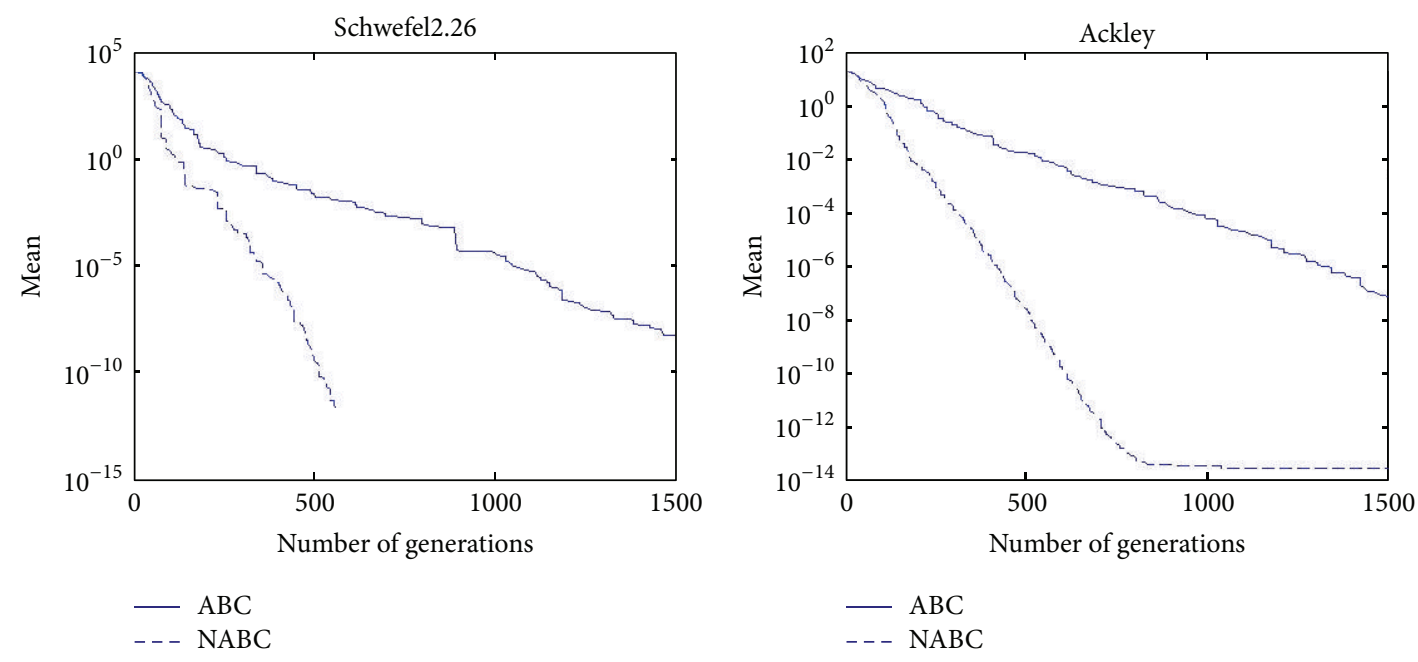

FIgURE 1: Convergence curves of $\mathrm{ABC}$ and NABC algorithms for $f_{1}-f_{10}$ functions $(D=30)$.

However, NABC still keeps fast convergence and best performance.

The results of NABC compared with the standard ABC algorithm are shown in Table 2 in terms of the best, worst, median, mean, and standard deviation of the solutions. These results show that the convergence rate of $\mathrm{NABC}$ is better than the standard $\mathrm{ABC}$ algorithm for most test functions. The rate of convergence obviously increases on $f_{1}-f_{3}, f_{5}$, $f_{7}-f_{11}$ functions from Figure 1 and Table 2. NABC can find the optimal solutions on functions $f_{5}, f_{7}, f_{8}$. The convergence rate of $\mathrm{NABC}$ is the same order of magnitude as the standard $\mathrm{ABC}$ algorithm on $f_{6}$. So the superiority of standard ABC algorithm is not very obvious. From the results in Table 2, NABC can obtain better and closer-to-optimal solutions than the standard $A B C$ algorithm for most of the functions. In a word, NABC increases the exploitation greatly. NABC also retains good exploration. Here, "+" indicates that NABC is statistically significantly better than its corresponding competitor algorithm; " $\approx$ " stands for that the result of the corresponding algorithm is statistically similar with that of NABC.

NABC is further compared with GABC [13] and EABC [15] in Tables 3 and 4. The results of GABC and EABC are gained from [15] directly. It is clear that NABC works better in almost all the cases and achieves better performance than GABC. The rate of convergence of NABC increases obviously on all functions in Tables 3 and 4 . The convergence rate of NABC is a little worse than that of EABC from Table 3. In particular, the convergence rate of Schwefel2.21 and Rosenbrock is better than EABC in Table 4.

At the same time, NABC is compared with four variants of DE (DE [17], jDE [18], JADE [19], and SaDE [20]) and five variants of PSO (FIPS [21], HPSO-TVAC [22], CLPSO [23], FPSO [24], OLPSO-G [25]). The results of DEs and PSOs are gained from [15] directly. The maximum number of function evaluations (FEs) is shown in Tables 5 and 6. Here, FEs is Maxgen $* \mathrm{SN}$. It can be seen that $\mathrm{NABC}$ is better on almost all the test functions except Ackley in JADE and OLPSO-G.
In Table 7, we can find the execution time of $\mathrm{ABC}$ is similar with that of NABC. So, NABC is largely faster than $\mathrm{ABC}$ and does not add the execution time.

\section{Application}

5.1. Knapsack Problems. We know that Knapsack problem [26] is an engineering optimization problem. It can be defined an unconstrained optimization problem and described as follows:

$$
\min f(x)=-\sum_{i=1}^{n} p_{i} x_{i}+\alpha * \max \left(0, \sum_{i=1}^{n} w_{i} x_{i}-c\right)
$$

where $w_{i}$ is each item's weight and $p_{i}$ is each item's profit, respectively. The knapsack has a limited weight capability of $c$. The objective function of this problem is to pack the knapsack so that the items in it have the maximal total profit. The decision variable $x_{i}$ is the value one if the item $i$ is packed; otherwise, it is the value zero. And we assume that all profits and weights are positive, and all weights are smaller than the capacity of $c$. $\alpha$ is a penalty factor which is set to $10 e-20$ in this paper. Tables 8 and 9 are parameters of five standard knapsack problems. The experiments tested on five benchmark 0-1 knapsack problems are performed to compare the performance of NABC with that of standard ABC. The maximum number of generations is set to 2000 . The population size is 100, namely, $\mathrm{SN}=50$, and Limit is 10 . Each of the experiments is repeated 30 times independently.

5.2. Data Analysis. In Table 10, we can see that NABC can easily solve the optimum of Knapsack problems. And it can find the optimum in most cases except $f_{5}$. ABC has the worst performance, because we can see that it can not find the optimums for $f_{2}$ and $f_{5}$. In short, NABC has a better performance than $\mathrm{ABC}$ on solving the five knapsack problems. 
TABLE 2: Best, worst, median, mean, and standard deviation values obtained by ABC and NABC through 30 independent runs on 11 functions.

\begin{tabular}{|c|c|c|c|c|c|c|c|c|}
\hline Function & Dim & & Best & Worst & Median & Mean & $\mathrm{SD}$ & Significant \\
\hline \multirow{6}{*}{$f_{1}$} & \multirow{2}{*}{30} & $\mathrm{ABC}$ & $5.04 e-16$ & $7.25 e-16$ & $5.04 e-16$ & $6.06 e-16$ & $9.34 e-17$ & \multirow{2}{*}{+} \\
\hline & & NABC & $1.13 e-55$ & $9.66 e-54$ & $6.66 e-55$ & $1.10 e-54$ & $1.71 e-54$ & \\
\hline & \multirow{2}{*}{60} & $\mathrm{ABC}$ & $1.40 e-15$ & $1.87 e-15$ & $1.52 e-15$ & $1.62 e-15$ & $2.02 e-16$ & \multirow{2}{*}{+} \\
\hline & & NABC & $1.03 e-53$ & $4.66 e-52$ & $9.06 e-53$ & $1.48 e-52$ & $1.37 e-52$ & \\
\hline & \multirow{2}{*}{100} & $\mathrm{ABC}$ & $2.20 e-15$ & $9.32 e-15$ & $5.21 e-15$ & $3.36 e-15$ & $2.01 e-15$ & \multirow{2}{*}{+} \\
\hline & & NABC & $1.61 e-52$ & $1.21 e-51$ & $3.69 e-51$ & $1.45 e-51$ & $8.91 e-52$ & \\
\hline \multirow{6}{*}{$f_{2}$} & \multirow{2}{*}{30} & $\mathrm{ABC}$ & $4.30 e-16$ & $6.34 e-16$ & $4.50 e-16$ & $5.03 e-16$ & $7.11 e-17$ & \multirow{2}{*}{+} \\
\hline & & NABC & $1.64 e-57$ & $7.82 e-56$ & $1.16 e-56$ & $2.70 e-56$ & $2.48 e-56$ & \\
\hline & \multirow{2}{*}{60} & $\mathrm{ABC}$ & $1.43 e-15$ & $2.00 e-15$ & $1.62 e-15$ & $1.67 e-15$ & $1.84 e-16$ & \multirow{2}{*}{+} \\
\hline & & NABC & $4.43 e-55$ & $7.82 e-53$ & $8.01 e-54$ & $2.08 e-53$ & $2.33 e-53$ & \\
\hline & \multirow{2}{*}{100} & $\mathrm{ABC}$ & $2.47 e-15$ & $3.66 e-15$ & $3.43 e-05$ & $3.19 e-15$ & $4.21 e-16$ & \multirow{2}{*}{+} \\
\hline & & NABC & $3.51 e-53$ & $5.75 e-52$ & $2.66 e-52$ & $3.01 e-52$ & $1.56 e-52$ & \\
\hline \multirow{6}{*}{$f_{3}$} & \multirow{2}{*}{30} & $\mathrm{ABC}$ & $2.38 e-11$ & $1.34 e-10$ & $6.42 e-11$ & $7.05 e-11$ & $5.19 e-11$ & \multirow{2}{*}{+} \\
\hline & & NABC & $5.06 e-30$ & $4.11 e-29$ & $1.73 e-29$ & $2.02 e-29$ & $1.10 e-29$ & \\
\hline & \multirow{2}{*}{60} & $\mathrm{ABC}$ & $9.02 e-11$ & $8.23 e-10$ & $5.12 e-10$ & $4.08 e-10$ & $1.57 e-10$ & \multirow{2}{*}{+} \\
\hline & & NABC & $7.12 e-29$ & $5.72 e-28$ & $2.57 e-28$ & $2.92 e-28$ & $1.54 e-28$ & \\
\hline & \multirow{2}{*}{100} & $\mathrm{ABC}$ & $2.05 e-09$ & $5.36 e-08$ & $4.84 e-09$ & $3.67 e-09$ & $4.80 e-09$ & + \\
\hline & & NABC & $2.85 e-28$ & $2.81 e-27$ & $7.35 e-28$ & $1.04 e-27$ & $6.88 e-28$ & \\
\hline & 30 & $\mathrm{ABC}$ & $8.68 e+00$ & $1.20 e+01$ & $1.84 e+01$ & $1.10 e+01$ & $1.29 e-00$ & + \\
\hline & & NABC & $1.77 e-01$ & $3.18 e-01$ & $2.68 e-01$ & $2.67 e-01$ & $3.55 e-02$ & $T$ \\
\hline$f_{4}$ & 60 & $\mathrm{ABC}$ & $4.53 e+01$ & $5.08 e+01$ & $5.02 e+01$ & $4.86 e+01$ & $1.90 e-00$ & + \\
\hline & 00 & NABC & $1.90 e+00$ & $3.16 e+00$ & $2.74 e+00$ & $2.71 e+00$ & $3.14 e-01$ & \\
\hline & 100 & $\mathrm{ABC}$ & $6.92 e+01$ & $7.43 e+01$ & $7.26 e+01$ & $7.25 e+01$ & $1.86 e-00$ & + \\
\hline & 100 & NABC & $8.32 e+00$ & $1.10 e+01$ & $9.79 e+00$ & $9.92 e+00$ & $7.04 e-01$ & \\
\hline & 30 & $\mathrm{ABC}$ & 0 & 0 & 0 & 0 & 0 & \\
\hline & 30 & NABC & 0 & 0 & 0 & 0 & 0 & $\approx$ \\
\hline$f_{5}$ & 60 & $\mathrm{ABC}$ & 0 & 0 & 0 & 0 & 0 & \\
\hline & & NABC & 0 & 0 & 0 & 0 & 0 & $\approx$ \\
\hline & 100 & $\mathrm{ABC}$ & 0 & 0 & 0 & 0 & 0 & \\
\hline & & NABC & 0 & 0 & 0 & 0 & 0 & $\approx$ \\
\hline & 30 & $\mathrm{ABC}$ & $8.12 e-03$ & $1.68 e-01$ & $1.33 e-02$ & $4.86 e-02$ & $4.84 e-02$ & $\approx$ \\
\hline & 30 & NABC & $2.21 e-03$ & $2.31 e-01$ & $3.32 e-02$ & $5.99 e-02$ & $5.64 e-02$ & \\
\hline$f_{6}$ & 60 & $\mathrm{ABC}$ & $1.08 e-02$ & $5.88 e-01$ & $1.56 e-01$ & $1.41 e-01$ & $1.65 e-01$ & \\
\hline & & NABC & $2.88 e-04$ & $3.88 e-01$ & $4.73 e-02$ & $1.02 e-01$ & $9.95 e-02$ & $\approx$ \\
\hline & 100 & $\mathrm{ABC}$ & $3.57 e-02$ & $9.94 e-01$ & $1.45 e-01$ & $2.97 e-01$ & $3.64 e-01$ & \\
\hline & & NABC & $2.92 e-04$ & $1.03 e+00$ & $2.16 e-01$ & $3.50 e-01$ & $3.28 e-01$ & $\approx$ \\
\hline & 30 & $\mathrm{ABC}$ & $5.36 e-15$ & $1.46 e-01$ & $4.56 e-09$ & $9.58 e-04$ & $1.05 e-03$ & + \\
\hline & & NABC & 0 & 0 & 0 & 0 & 0 & \\
\hline$f_{-}$ & 60 & $\mathrm{ABC}$ & $5.87 e-12$ & $1.99 e-00$ & $1.25 e-09$ & $2.68 e-02$ & $6.32 e-02$ & + \\
\hline$J_{7}$ & & NABC & 0 & 0 & 0 & 0 & 0 & + \\
\hline & 100 & $\mathrm{ABC}$ & $8.65 e-13$ & $1.99 e-00$ & $1.15 e-06$ & $4.97 e-02$ & $2.16 e-01$ & + \\
\hline & 100 & NABC & 0 & 0 & 0 & 0 & 0 & + \\
\hline & & $\mathrm{ABC}$ & $8.01 e-15$ & $1.30 e-12$ & $8.14 e-13$ & $2.37 e-13$ & $4.18 e-13$ & + \\
\hline & 30 & NABC & 0 & 0 & 0 & 0 & 0 & + \\
\hline$f_{0}$ & 60 & $\mathrm{ABC}$ & $8.32 e-14$ & $1.34 e-11$ & $7.20 e-13$ & $3.45 e-12$ & $2.89 e-12$ & + \\
\hline$J_{8}$ & 60 & NABC & 0 & 0 & 0 & 0 & 0 & + \\
\hline & 100 & $\mathrm{ABC}$ & $5.36 e-14$ & $1.58 e-09$ & $4.27 e-10$ & $1.80 e-10$ & $2.03 e-10$ & + \\
\hline & 100 & NABC & 0 & 0 & 0 & 0 & 0 & \\
\hline
\end{tabular}


TABLE 2: Continued.

\begin{tabular}{|c|c|c|c|c|c|c|c|c|}
\hline Function & Dim & & Best & Worst & Median & Mean & SD & Significant \\
\hline \multirow{6}{*}{$f_{9}$} & \multirow{2}{*}{30} & $\mathrm{ABC}$ & $1.54 e-06$ & $2.37 e+02$ & $3.76 e-01$ & $8.86 e+01$ & $8.62 e+01$ & \multirow{2}{*}{+} \\
\hline & & NABC & $-3.64 e-12$ & $-1.82 e-12$ & $-1.82 e-12$ & $-2.00 e-12$ & $5.55 e-13$ & \\
\hline & \multirow{2}{*}{60} & $\mathrm{ABC}$ & $3.55 e+02$ & $7.69 e+02$ & $7.69 e+02$ & $5.40 e+02$ & $1.41 e+02$ & \multirow{2}{*}{+} \\
\hline & & NABC & $2.91 e-11$ & $3.64 e-11$ & $3.64 e-11$ & $3.54 e-11$ & $2.52 e-12$ & \\
\hline & \multirow{2}{*}{100} & $\mathrm{ABC}$ & $7.81 e+02$ & $1.55 e+03$ & $1.51 e+03$ & $1.29 e+03$ & $2.23 e+02$ & \multirow{2}{*}{+} \\
\hline & & NABC & $1.02 e-10$ & $1.16 e-10$ & $1.09 e-10$ & $1.10 e-10$ & $4.58 e-12$ & \\
\hline \multirow{6}{*}{$f_{10}$} & \multirow{2}{*}{30} & $\mathrm{ABC}$ & $5.36 e-10$ & $1.23 e-08$ & $3.67 e-09$ & $1.45 e-09$ & $2.37 e-09$ & \multirow{2}{*}{+} \\
\hline & & NABC & $2.22 e-14$ & $2.93 e-14$ & $2.93 e-14$ & $2.66 e-14$ & $3.32 e-15$ & \\
\hline & \multirow{2}{*}{60} & $\mathrm{ABC}$ & $8.14 e-09$ & $7.54 e-08$ & $5.38 e-08$ & $4.60 e-08$ & $2.04 e-08$ & \multirow{2}{*}{+} \\
\hline & & NABC & $5.06 e-14$ & $6.84 e-14$ & $6.48 e-14$ & $6.44 e-14$ & $5.26 e-15$ & \\
\hline & \multirow{2}{*}{100} & $\mathrm{ABC}$ & $5.35 e-08$ & $4.28 e-07$ & $1.04 e-07$ & $2.83 e-07$ & $1.57 e-07$ & \multirow{2}{*}{+} \\
\hline & & NABC & $1.04 e-13$ & $1.22 e-13$ & $1.15 e-13$ & $1.16 e-13$ & $4.75 e-15$ & \\
\hline \multirow{6}{*}{$f_{11}$} & \multirow{2}{*}{150} & $\mathrm{ABC}$ & -77.4221 & -76.9602 & -77.0460 & -77.1658 & $2.11 e-01$ & \multirow{2}{*}{+} \\
\hline & & NABC & -78.3323 & -78.3323 & -78.3323 & -78.3323 & $1.45 e-14$ & \\
\hline & \multirow{2}{*}{300} & $\mathrm{ABC}$ & -77.0391 & -76.8430 & -77.0391 & -76.9258 & $6.76 e-02$ & \multirow{2}{*}{+} \\
\hline & & NABC & -78.3323 & -78.3323 & -78.3323 & -78.3323 & $1.45 e-14$ & \\
\hline & \multirow{2}{*}{500} & $\mathrm{ABC}$ & -76.8562 & -76.5849 & -76.8562 & -76.7220 & $9.52 e-02$ & \multirow{2}{*}{+} \\
\hline & & NABC & -78.3323 & -78.3323 & -78.3323 & -78.3323 & $1.45 e-14$ & \\
\hline
\end{tabular}

TABLE 3: Comparison among GABC, EABC, and NABC on optimizing 8 benchmark functions.

\begin{tabular}{|c|c|c|c|c|c|c|c|c|}
\hline \multirow{2}{*}{ Function } & \multirow{2}{*}{ Max.FEs } & \multirow{2}{*}{ Dim } & \multicolumn{2}{|c|}{ GABC } & \multicolumn{2}{|c|}{ EABC } & \multicolumn{2}{|c|}{ NABC } \\
\hline & & & Mean & SD & Mean & $\mathrm{SD}$ & Mean & SD \\
\hline \multirow{3}{*}{ Sphere } & 150,000 & 30 & $1.37 e-25$ & $2.70 e-25$ & $4.42 e-67$ & $2.71 e-67$ & $1.10 e-54$ & $1.71 e-54$ \\
\hline & 300,000 & 60 & $4.86 e-23$ & $5.14 e-23$ & $2.30 e-64$ & $1.03 e-64$ & $1.48 e-52$ & $1.37 e-52$ \\
\hline & 500,000 & 100 & $9.05 e-22$ & $4.28 e-22$ & $6.37 e-63$ & $2.55 e-63$ & $1.45 e-51$ & $8.91 e-52$ \\
\hline \multirow{3}{*}{ Schwefel2.22 } & 150,000 & 30 & $5.56 e-15$ & $9.79 e-15$ & $5.51 e-35$ & $7.02 e-35$ & $2.02 e-29$ & $1.10 e-29$ \\
\hline & 300,000 & 60 & $5.61 e-14$ & $9.93 e-15$ & $5.08 e-33$ & $1.95 e-34$ & $2.92 e-28$ & $1.54 e-28$ \\
\hline & 500,000 & 100 & $2.29 e-13$ & $3.97 e-13$ & $4.68 e-32$ & $1.42 e-32$ & $1.04 e-27$ & $6.88 e-28$ \\
\hline \multirow{3}{*}{ Schwefel2.21 } & 150,000 & 30 & $3.07 e-00$ & $4.72 e-01$ & $6.46 e-01$ & $1.03 e-01$ & $2.67 e-01$ & $3.55 e-02$ \\
\hline & 300,000 & 60 & $3.85 e+01$ & $3.42 e-00$ & $2.49 e+01$ & $2.04 e-00$ & $2.71 e+00$ & $3.14 e-01$ \\
\hline & 500,000 & 100 & $7.04 e+01$ & $1.20 e-00$ & $6.19 e+01$ & $1.24 e-00$ & $9.92 e+00$ & $7.04 e-01$ \\
\hline \multirow{3}{*}{ Rosenbrock } & 150,000 & 30 & $1.30 e-00$ & $1.64 e-00$ & $8.67 e-02$ & $7.48 e-02$ & $5.99 e-02$ & $5.64 e-02$ \\
\hline & 300,000 & 60 & $1.66 e+01$ & $3.28 e+01$ & $2.03 e-01$ & $1.32 e-01$ & $1.02 e-01$ & $9.95 e-02$ \\
\hline & 500,000 & 100 & $2.39 e+01$ & $3.34 e+01$ & $5.03 e-01$ & $8.82 e-01$ & $3.50 e-01$ & $3.28 e-01$ \\
\hline \multirow{3}{*}{ Griewank } & 100,000 & 30 & $1.40 e-08$ & $1.16 e-08$ & 0 & 0 & 0 & 0 \\
\hline & 150,000 & 60 & $1.58 e-06$ & $1.00 e-06$ & 0 & 0 & 0 & 0 \\
\hline & 250,000 & 100 & $2.44 e-06$ & $2.50 e-06$ & 0 & 0 & 0 & 0 \\
\hline \multirow{3}{*}{ Rastrigin } & 100,000 & 30 & $7.08 e-03$ & $1.19 e-03$ & 0 & 0 & 0 & 0 \\
\hline & 150,000 & 60 & $3.47 e-00$ & $8.78 e-01$ & 0 & 0 & 0 & 0 \\
\hline & 250,000 & 100 & $9.78 e-00$ & $2.91 e-00$ & 0 & 0 & 0 & 0 \\
\hline \multirow{3}{*}{ Schwefel2.26 } & 50,000 & 30 & $6.38 e-00$ & $2.38 e+01$ & 0 & 0 & $1.71 e-09$ & $3.01 e-10$ \\
\hline & 100,000 & 60 & $3.30 e+01$ & $4.94 e+01$ & $3.92 e-11$ & $3.56 e-12$ & $1.24 e-09$ & $5.02 e-09$ \\
\hline & 200,000 & 100 & $6.05 e+01$ & $8.05 e+01$ & $1.12 e-10$ & $3.56 e-12$ & $1.22 e-10$ & $2.97 e-11$ \\
\hline \multirow{3}{*}{ Ackley } & 50,000 & 30 & $6.45 e-03$ & $2.68 e-03$ & $3.39 e-10$ & $6.95 e-11$ & $2.04 e-08$ & $4.72 e-09$ \\
\hline & 100,000 & 60 & $2.96 e-03$ & $2.14 e-03$ & $1.81 e-09$ & $2.70 e-10$ & $4.45 e-08$ & $1.50 e-08$ \\
\hline & 150,000 & 100 & $6.01 e-02$ & $9.60 e-02$ & $4.53 e-08$ & $1.23 e-08$ & $6.01 e-07$ & $3.23 e-07$ \\
\hline
\end{tabular}


TABLE 4: Comparison among ABC, EABC, and NABC on optimizing 4 benchmark functions with $D=30,60,100$.

\begin{tabular}{|c|c|c|c|c|c|c|c|}
\hline \multirow{2}{*}{ Function } & \multirow{2}{*}{ Dim } & \multicolumn{2}{|c|}{$\mathrm{ABC}$} & \multicolumn{2}{|c|}{ EABC } & \multicolumn{2}{|c|}{ NABC } \\
\hline & & Mean & $\mathrm{SD}$ & Mean & $\mathrm{SD}$ & Mean & SD \\
\hline \multirow{3}{*}{ Schwefel2.21 } & 30 & $1.10 e+01$ & $1.29 e-00$ & $6.46 e-01$ & $1.03 e-01$ & $2.67 e-01$ & $3.55 e-02$ \\
\hline & 60 & $4.86 e+01$ & $1.90 e-00$ & $2.49 e+01$ & $2.04 e-00$ & $2.71 e+00$ & $3.14 e-01$ \\
\hline & 100 & $7.25 e+01$ & $1.86 e-00$ & $6.19 e+01$ & $1.24 e-00$ & $9.92 e+00$ & $7.04 e-01$ \\
\hline \multirow{3}{*}{ Rosenbrock } & 30 & $4.86 e-02$ & $4.84 e-02$ & $8.67 e-02$ & $7.48 e-02$ & $5.99 e-02$ & $5.64 e-02$ \\
\hline & 60 & $1.41 e-01$ & $1.65 e-01$ & $2.03 e-01$ & $1.32 e-01$ & $1.02 e-01$ & $9.95 e-02$ \\
\hline & 100 & $2.97 e-01$ & $3.64 e-01$ & $5.03 e-01$ & $8.82 e-01$ & $3.50 e-01$ & $3.28 e-01$ \\
\hline \multirow{3}{*}{ Schwefel2.26 } & 30 & $8.86 e+01$ & $8.62 e+01$ & $-1.23 e-13$ & $1.09 e-13$ & $-2.00 e-12$ & $5.55 e-13$ \\
\hline & 60 & $5.40 e+02$ & $1.41 e+02$ & $2.91 e-11$ & 0 & $3.54 e-11$ & $2.52 e-12$ \\
\hline & 100 & $1.29 e+03$ & $2.23 e+02$ & $9.45 e-11$ & 0 & $1.10 e-10$ & $4.58 e-12$ \\
\hline \multirow{3}{*}{ Ackley } & 30 & $1.45 e-09$ & $2.37 e-09$ & $1.36 e-14$ & $1.74 e-15$ & $2.66 e-14$ & $3.32 e-15$ \\
\hline & 60 & $4.60 e-08$ & $2.04 e-08$ & $4.49 e-14$ & $2.84 e-15$ & $6.44 e-14$ & $5.26 e-15$ \\
\hline & 100 & $2.83 e-07$ & $1.57 e-07$ & $9.53 e-14$ & $2.84 e-15$ & $1.16 e-13$ & $4.75 e-15$ \\
\hline
\end{tabular}

TABLE 5: Comparison among NABC, DE, jDE, JADE, and SaDE on optimizing 8 benchmark functions with $D=30$.

\begin{tabular}{|c|c|c|c|c|c|c|c|}
\hline Function & Max.FEs & & $\mathrm{DE}$ & $\mathrm{jDE}$ & JADE & $\mathrm{SaDE}$ & NABC \\
\hline \multirow{2}{*}{ Sphere } & \multirow{2}{*}{150,000} & Mean & $9.8 e-14$ & $1.46 e-28$ & $1.32 e-54$ & $3.28 e-20$ & $1.10 e-54$ \\
\hline & & $\mathrm{SD}$ & $8.4 e-14$ & $1.78 e-28$ & $9.22 e-54$ & $3.62 e-20$ & $1.71 e-54$ \\
\hline \multirow{2}{*}{ Schwefel2.22 } & \multirow{2}{*}{200,000} & Mean & $1.6 e-09$ & $9.02 e-24$ & $3.18 e-25$ & $3.51 e-25$ & $7.20 e-40$ \\
\hline & & $\mathrm{SD}$ & $1.1 e-09$ & $6.01 e-24$ & $2.05 e-25$ & $2.74 e-25$ & $3.33 e-40$ \\
\hline \multirow{2}{*}{ Step } & \multirow{2}{*}{10,000} & Mean & $4.7 e+03$ & $6.13 e+02$ & $5.62 e+00$ & $5.07 e+01$ & 0 \\
\hline & & $\mathrm{SD}$ & $1.1 e+03$ & $1.72 e+02$ & $1.87 e+00$ & $1.34 e+01$ & 0 \\
\hline \multirow{2}{*}{ Rosenbrock } & \multirow{2}{*}{300,000} & Mean & $2.1 e+00$ & $1.3 e+01$ & $3.2 e-01$ & $2.1 e+01$ & $4.22 e-02$ \\
\hline & & $\mathrm{SD}$ & $1.5 e+00$ & $1.4 e+01$ & $1.1 e+00$ & $7.8 e+00$ & $4.93 e-02$ \\
\hline \multirow{2}{*}{ Griewank } & \multirow{2}{*}{50,000} & Mean & $2.0 e-01$ & $7.29 e-06$ & $1.57 e-08$ & $2.52 e-09$ & $1.09 e-08$ \\
\hline & & $\mathrm{SD}$ & $1.1 e-01$ & $1.05 e-05$ & $1.09 e-07$ & $1.24 e-08$ & $2.86 e-08$ \\
\hline \multirow{2}{*}{ Rastrigin } & \multirow{2}{*}{100,000} & Mean & $1.8 e+02$ & $3.32 e-04$ & $1.33 e-01$ & $2.43 e+00$ & 0 \\
\hline & & $\mathrm{SD}$ & $1.3 e+01$ & $6.39 e-04$ & $9.74 e-02$ & $1.60 e+00$ & 0 \\
\hline \multirow{2}{*}{ Ackley } & \multirow{2}{*}{50,000} & Mean & $1.1 e-01$ & $2.37 e-04$ & $3.35 e-09$ & $3.81 e-06$ & $2.04 e-08$ \\
\hline & & $\mathrm{SD}$ & $3.9 e-02$ & $7.10 e-05$ & $2.84 e-09$ & $8.26 e-07$ & $4.72 e-09$ \\
\hline \multirow{2}{*}{ Schwefel2.26 } & \multirow{2}{*}{100,000} & Mean & $5.9 e+03$ & $1.70 e-10$ & $2.62 e-04$ & $1.13 e-08$ & $-1.94 e-12$ \\
\hline & & SD & $1.1 e+03$ & $1.71 e-10$ & $3.59 e-04$ & $1.08 e-08$ & $4.61 e-13$ \\
\hline
\end{tabular}

TABLE 6: Comparison among NABC, FIPS, HPSO-TVAC, CLPSO, FPSO, and OLPSO-G on optimizing 8 benchmark functions with 200,000 FEs.

\begin{tabular}{|c|c|c|c|c|c|c|c|}
\hline Function & & FIPS & HPSO-TVAC & CLPSO & FPSO & OLPSO-G & NABC \\
\hline \multirow{2}{*}{ Sphere } & Mean & $2.42 e-13$ & $2.83 e-33$ & $1.58 e-12$ & $2.40 e-16$ & $4.12 e-54$ & $5.45 e-75$ \\
\hline & $\mathrm{SD}$ & $1.73 e-13$ & $3.19 e-33$ & $7.70 e-13$ & $2.00 e-31$ & $6.34 e-54$ & $3.55 e-75$ \\
\hline \multirow{2}{*}{ Schwefel2.22 } & Mean & $2.76 e-08$ & $9.03 e-20$ & $2.51 e-08$ & $1.58 e-11$ & $9.85 e-30$ & $7.20 e-40$ \\
\hline & SD & $9.04 e-09$ & $9.58 e-20$ & $5.84 e-09$ & $1.03 e-22$ & $1.01 e-29$ & $3.33 e-40$ \\
\hline \multirow{2}{*}{ Step } & Mean & 0 & 0 & 0 & 0 & 0 & 0 \\
\hline & SD & 0 & 0 & 0 & 0 & 0 & 0 \\
\hline \multirow{2}{*}{ Rosenbrock } & Mean & $2.51 e+01$ & $2.39 e+01$ & $1.13 e+01$ & $2.81 e+01$ & $2.15 e+01$ & $6.33 e-02$ \\
\hline & SD & $5.10 e-01$ & $2.65 e+01$ & $9.85 e-00$ & $2.31 e+02$ & $2.99 e+01$ & $6.34 e-02$ \\
\hline \multirow{2}{*}{ Griewank } & Mean & $9.01 e-12$ & $9.75 e-03$ & $9.02 e-09$ & $1.47 e-03$ & $4.83 e-03$ & 0 \\
\hline & SD & $1.84 e-11$ & $8.33 e-03$ & $8.57 e-09$ & $1.28 e-05$ & $8.63 e-03$ & 0 \\
\hline \multirow{2}{*}{ Rastrigin } & Mean & $6.51 e+01$ & $9.43 e-00$ & $9.09 e-05$ & $7.38 e+01$ & $1.07 e-00$ & 0 \\
\hline & $\mathrm{SD}$ & $1.33 e+01$ & $3.48 e-00$ & $1.25 e-04$ & $3.70 e+02$ & $9.92 e-01$ & 0 \\
\hline \multirow{2}{*}{ Ackley } & Mean & $2.33 e-07$ & $7.29 e-14$ & $3.66 e-07$ & $2.17 e-09$ & $7.98 e-15$ & $2.78 e-14$ \\
\hline & $\mathrm{SD}$ & $7.19 e-08$ & $3.00 e-14$ & $7.57 e-08$ & $1.71 e-18$ & $2.03 e-15$ & $2.59 e-15$ \\
\hline \multirow{2}{*}{ Schwefel2.26 } & Mean & $9.93 e+02$ & $1.59 e+03$ & $3.82 e-04$ & $1.34 e+03$ & $3.84 e+02$ & $-2.43 e-12$ \\
\hline & $\mathrm{SD}$ & $5.09 e+02$ & $3.26 e+02$ & $1.28 e-05$ & $2.77 e+02$ & $2.17 e+02$ & $8.72 e-13$ \\
\hline
\end{tabular}


TABLE 7: Execution time of ABC and NABC algorithm with $D=30,60,100$ (second).

\begin{tabular}{cccccccccccc}
\hline Dim & Algorithm & $f_{1}$ & $f_{2}$ & $f_{3}$ & $f_{4}$ & $f_{5}$ & $f_{6}$ & $f_{7}$ & $f_{8}$ & $f_{9}$ & $f_{10}$ \\
\hline \multirow{2}{*}{30} & ABC & 19.850 & 25.535 & 33.363 & 18.768 & 3.969 & 32.338 & 40.942 & 78.680 & 94.332 & 47.626 \\
& NABC & 17.661 & 25.697 & 36.352 & 20.056 & 2.768 & 30.612 & 18.229 & 31.331 & 77.497 & 42.660 \\
\hline \multirow{2}{*}{60} & ABC & 43.236 & 72.503 & 104.475 & 47.454 & 12.304 & 81.891 & 185.687 & 259.944 & 322.573 & 205.303 \\
& NABC & 44.900 & 73.449 & 108.912 & 47.991 & 8.202 & 80.281 & 95.126 & 129.497 & 265.051 & 186.870 \\
\hline \multirow{2}{*}{100} & ABC & 89.402 & 163.012 & 255.115 & 92.929 & 37.074 & 180.789 & 371.414 & 574.304 & 841.482 & 410.198 \\
& NABC & 88.507 & 168.964 & 255.134 & 91.418 & 21.081 & 175.828 & 334.624 & 308.107 & 687.463 & 365.939 \\
\hline
\end{tabular}

TABLE 8: The five benchmark knapsack problems.

\begin{tabular}{|c|c|c|}
\hline$f$ & Dim & Parameter $(w, p, c)$ \\
\hline$f_{1}$ & 10 & $w=(95,4,60,32,23,72,80,62,65,46) ; p=(55,10,47,5,4,50,8,61,85,87) ; c=269$ \\
\hline$f_{2}$ & 20 & $\begin{array}{l}w=(92,4,43,83,84,68,92,82,6,44,32,18,56,83,25,96,70,48,14,58) ; p=(44,46,90,72,91,40,75,35,8,54,78,40 \\
77,15,61,17,75,29,75,63) ; c=878\end{array}$ \\
\hline$f_{3}$ & 4 & $w=(6,5,9,7) ; p=(9,11,13,15) ; c=20$ \\
\hline$f_{4}$ & 4 & $w=(2,4,6,7) ; p=(6,10,12,13) ; c=11$ \\
\hline$f_{5}$ & 15 & $\begin{array}{l}w=(56.358531,80.874050,47.987304,89.596240,74.660482,85.894345,51.353496,1.498459,36.445204,16.589862 \\
44.569231,0.466933,37.788018,57.118442,60.716575) ; p=(0.125126,19.330424,58.500931,35.029145,82.284005 \\
17.410810,71.050142,30.399487,9.140294,14.731285,98.852504,11.908322,0.891140,53.166295,60.176397) ; c=375\end{array}$ \\
\hline
\end{tabular}

TABLE 9: The optimal solutions of the five benchmark knapsack problems.

\begin{tabular}{lccc}
\hline$f$ & Optimal solution & Optimal value & Value of constraint \\
\hline$f_{1}$ & $(0,1,1,1,0,0,0,1,1,1)$ & 295 & 0 \\
$f_{2}$ & $(1,1,1,1,1,1,1,1,1,1,1,1,1,0,1,0,1,0,1,1)$ & 1024 & -7 \\
$f_{3}$ & $(1,1,0,1)$ & 35 & -2 \\
$f_{4}$ & $(0,1,0,1)$ & 23 & 0 \\
$f_{5}$ & $(0,0,1,0,1,0,1,1,0,1,1,1,0,1,1)$ & 481.0694 & -20.0392 \\
\hline
\end{tabular}

TABLE 10: Best, worst, median, mean, and standard deviation values obtained by ABC and NABC.

\begin{tabular}{|c|c|c|c|c|c|c|}
\hline$f$ & Algorithm & Best & Worst & Median & Mean & SD \\
\hline \multirow{2}{*}{$f_{1}$} & $\mathrm{ABC}$ & 295 & 295 & 295 & 295 & 0 \\
\hline & NDABC & 295 & 295 & 295 & 295 & 0 \\
\hline \multirow{2}{*}{$f_{2}$} & $\mathrm{ABC}$ & 1024 & 1013 & 1024 & 1021 & 4.52 \\
\hline & NDABC & 1024 & 1024 & 1024 & 1024 & 0 \\
\hline \multirow{2}{*}{$f_{3}$} & $\mathrm{ABC}$ & 35 & 35 & 35 & 35 & 0 \\
\hline & NDABC & 35 & 35 & 35 & 35 & 0 \\
\hline \multirow{2}{*}{$f_{4}$} & $\mathrm{ABC}$ & 23 & 23 & 23 & 23 & 0 \\
\hline & NDABC & 23 & 23 & 23 & 23 & 0 \\
\hline \multirow{2}{*}{$f_{5}$} & $\mathrm{ABC}$ & 481.069 & 437.935 & 475.478 & 460.729 & 18.47 \\
\hline & NDABC & 481.069 & 435.786 & 481.069 & 470.771 & 8.79 \\
\hline
\end{tabular}

\section{Conclusion}

In this paper, we develop a novel artificial bee colony algorithm named NABC. We add the global best solution into the search equation to drive the new candidate solution only around the global best solution in order to improve the exploitation. And the search equation of the employed bees is improved to keep the exploration of algorithm. The experimental results tested on 11 benchmark functions show that the convergence of NABC is much faster than that of other algorithms, and the computing is more effective. At the same time, we apply NABC on solving five standard Knapsack problems and get good optimums. So it is fitted to solve many engineering practical problems.

\section{Conflict of Interests}

The authors declare that there is no conflict of interests regarding the publication of this paper. 


\section{Acknowledgments}

The authors are grateful to the editor and the anonymous reviewers for their valuable comments and suggestions.

\section{References}

[1] K. S. Tang, K. F. Man, S. Kwong, and Q. He, "Genetic algorithms and their applications," IEEE Signal Processing Magazine, vol. 13, no. 6 , pp. 22-37, 1996.

[2] J. Kennedy and R. Eberhart, "Particle swarm optimization," in Proceedings of the IEEE International Conference on Neural Networks, pp. 1942-1948, Perth, Australia, December 1995.

[3] M. Dorigo and L. M. Gambardella, "Ant colony system: a cooperative learning approach to the traveling salesman problem," IEEE Transactions on Evolutionary Computation, vol. 1, no. 1, pp. 53-66, 1997.

[4] D. Simon, "Biogeography-based optimization," IEEE Transactions on Evolutionary Computation, vol. 12, no. 6, pp. 702-713, 2008.

[5] D. Karaboga, "An idea based on honey bee swarm for numerical optimization,” Tech. Rep. TR06, Erciyes University, Kayseri, Turkey, 2005.

[6] B. Basturk and D. Karaboga, "An artificial bee colony (ABC) algorithm for numeric function optimization," in Proceedings of the IEEE Swarm Intelligence Symposium, May 2006.

[7] D. Karaboga and B. Basturk, "A powerful and efficient algorithm for numerical function optimization: artificial bee colony (ABC) algorithm," Journal of Global Optimization, vol. 39, no. 3, pp. 459-471, 2007.

[8] D. Karaboga and B. Basturk, "On the performance of artificial bee colony (ABC) algorithm," Applied Soft Computing Journal, vol. 8, no. 1, pp. 687-697, 2008.

[9] D. Karaboga and B. Akay, "A comparative study of artificial Bee colony algorithm," Applied Mathematics and Computation, vol. 214, no. 1, pp. 108-132, 2009.

[10] D. Karaboga, B. Akay, and C. Ozturk, "Artificial bee colony (ABC) optimization algorithm for training feed-forward neural networks," in Modeling Decisions for Artificial Intelligence, vol. 4617 of Lecture Notes in Computer Science, pp. 318-329, Springer, Berlin, Germany, 2007.

[11] Y.-F. Liu and S.-Y. Liu, "A hybrid discrete artificial bee colony algorithm for permutation flowshop scheduling problem," Applied Soft Computing Journal, vol. 13, no. 3, pp. 1459-1463, 2013.

[12] D. Karaboga and B. Basturk, "Artificial bee colony (ABC) optimization algorithm for solving constrained optimization problems," in Foundations of Fuzzy Logic and Soft Computing, vol. 4529 of Lecture Notes in Computer Science, pp. 789-798, Springer, Berlin, Germany, 2007.

[13] G. P. Zhu and S. Kwong, "Gbest-guided artificial bee colony algorithm for numerical function optimization," Applied Mathematics and Computation, vol. 217, no. 7, pp. 3166-3173, 2010.

[14] B. Basturk and D. Karaboga, "A modified artificial bee colony algorithm for real-parameter optimization," Information Sciences, vol. 192, pp. 120-142, 2012.

[15] W.-F. Gao, S.-Y. Liu, and L.-L. Huang, "Enhancing artificial bee colony algorithm using more information-based search equations," Information Sciences, vol. 270, pp. 112-133, 2014.

[16] D. Karaboga and B. Gorkemli, "A quick artificial bee colony (qABC) algorithm and its performance on optimization problems," Applied Soft Computing, vol. 23, pp. 227-238, 2014.
[17] R. Storn and K. Price, "Differential evolution-a simple and efficient heuristic for global optimization over continuous spaces," Journal of Global Optimization, vol. 23, pp. 689-694, 2010.

[18] J. Brest, S. Greiner, B. Bošković, M. Mernik, and V. Zumer, "Self-adapting control parameters in differential evolution: a comparative study on numerical benchmark problems," IEEE Transactions on Evolutionary Computation, vol. 10, no. 6, pp. 646-657, 2006.

[19] J. Zhang and A. C. Sanderson, "JADE: adaptive differential evolution with optional external archive," IEEE Transactions on Evolutionary Computation, vol. 13, no. 5, pp. 945-958, 2009.

[20] A. K. Qin, V. L. Huang, and P. N. Suganthan, "Differential evolution algorithm with strategy adaptation for global numerical optimization," IEEE Transactions on Evolutionary Computation, vol. 13, no. 2, pp. 398-417, 2008.

[21] R. Mendes, J. Kennedy, and J. Neves, "The fully informed particle swarm: simpler, maybe better," IEEE Transactions on Evolutionary Computation, vol. 8, no. 3, pp. 204-210, 2004.

[22] A. Ratnaweera, S. K. Halgamuge, and H. C. Watson, "Selforganizing hierarchical particle swarm optimizer with timevarying acceleration coefficients," IEEE Transactions on Evolutionary Computation, vol. 8, no. 3, pp. 240-255, 2004.

[23] J. J. Liang, A. K. Qin, P. N. Suganthan, and S. Baskar, "Comprehensive learning particle swarm optimizer for global optimization of multimodal functions," IEEE Transactions on Evolutionary Computation, vol. 10, no. 3, pp. 281-295, 2006.

[24] M. A. M. de Oca, T. Stützle, M. Birattari, and M. Dorigo, "Frankenstein's PSO: a composite particle swarm optimization algorithm," IEEE Transactions on Evolutionary Computation, vol. 13, no. 5, pp. 1120-1132, 2009.

[25] Z.-H. Zhan, J. Zhang, Y. Li, and Y.-H. Shi, "Orthogonal learning particle swarm optimization," IEEE Transactions on Evolutionary Computation, vol. 15, no. 6, pp. 832-847, 2011.

[26] D. Zou, L. Gao, S. Li, and J. Wu, "Solving 0-1 knapsack problem by a novel global harmony search algorithm," Applied Soft Computing Journal, vol. 11, no. 2, pp. 1556-1564, 2011. 


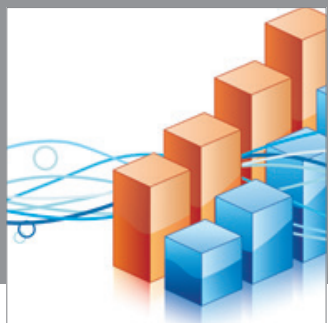

Advances in

Operations Research

mansans

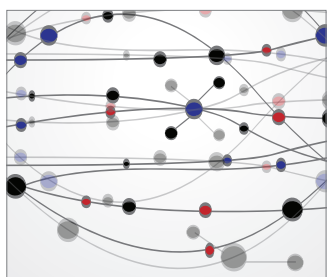

The Scientific World Journal
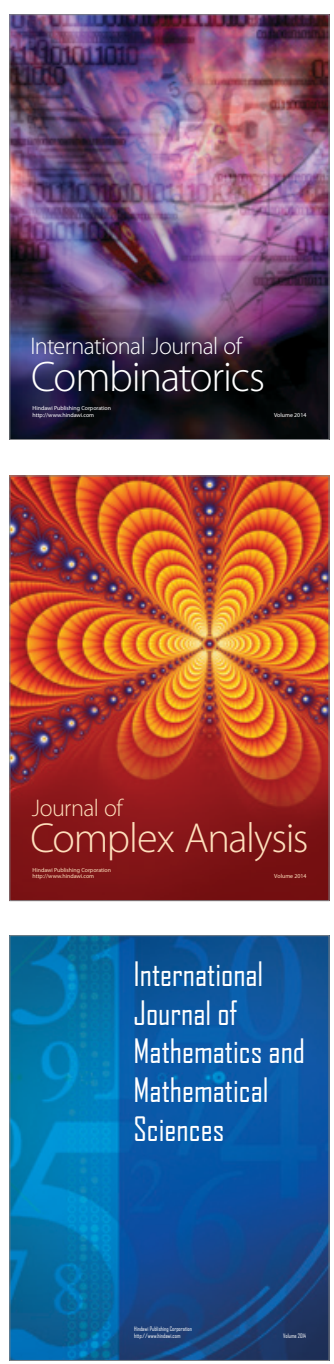
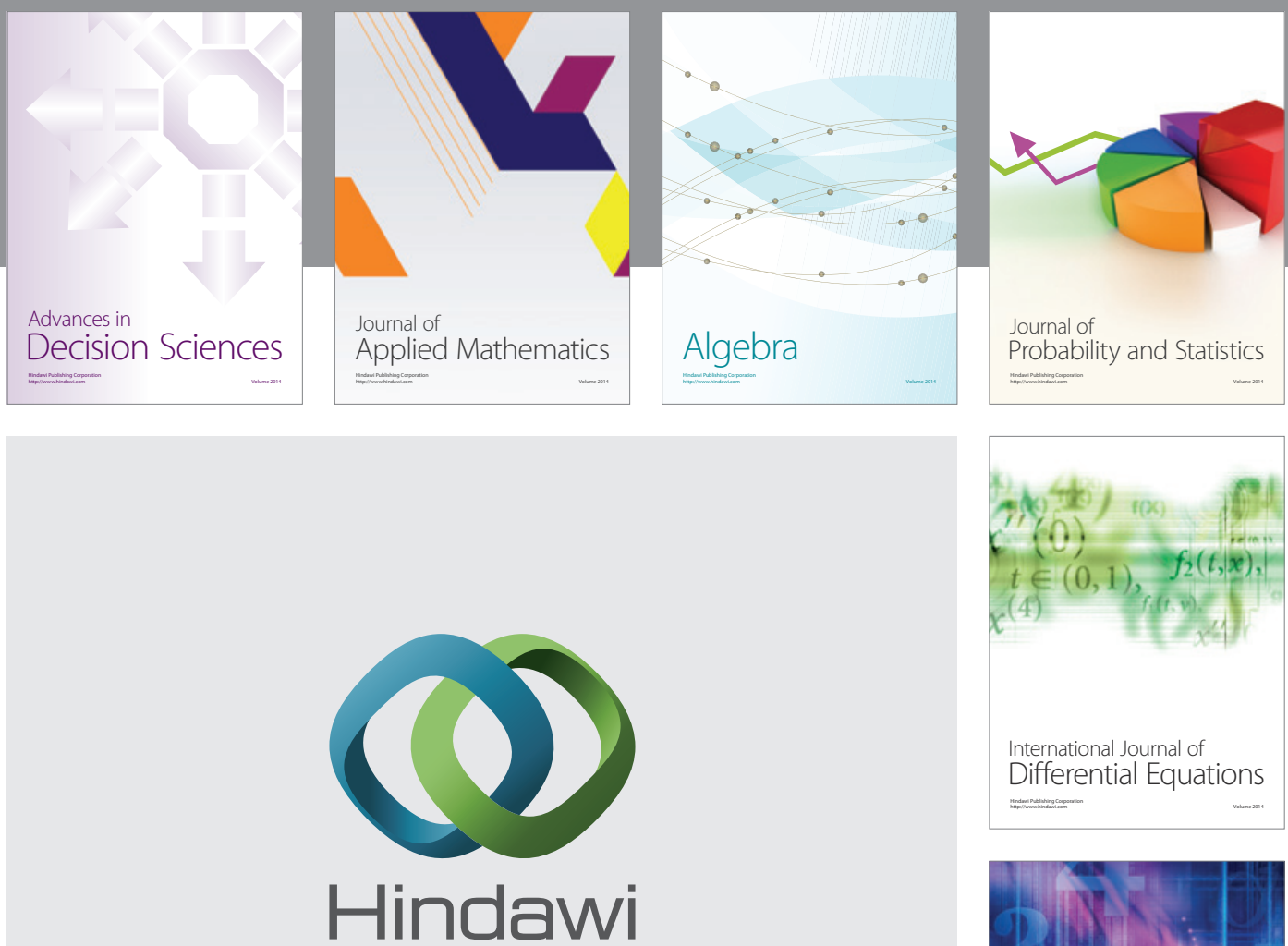

Submit your manuscripts at http://www.hindawi.com
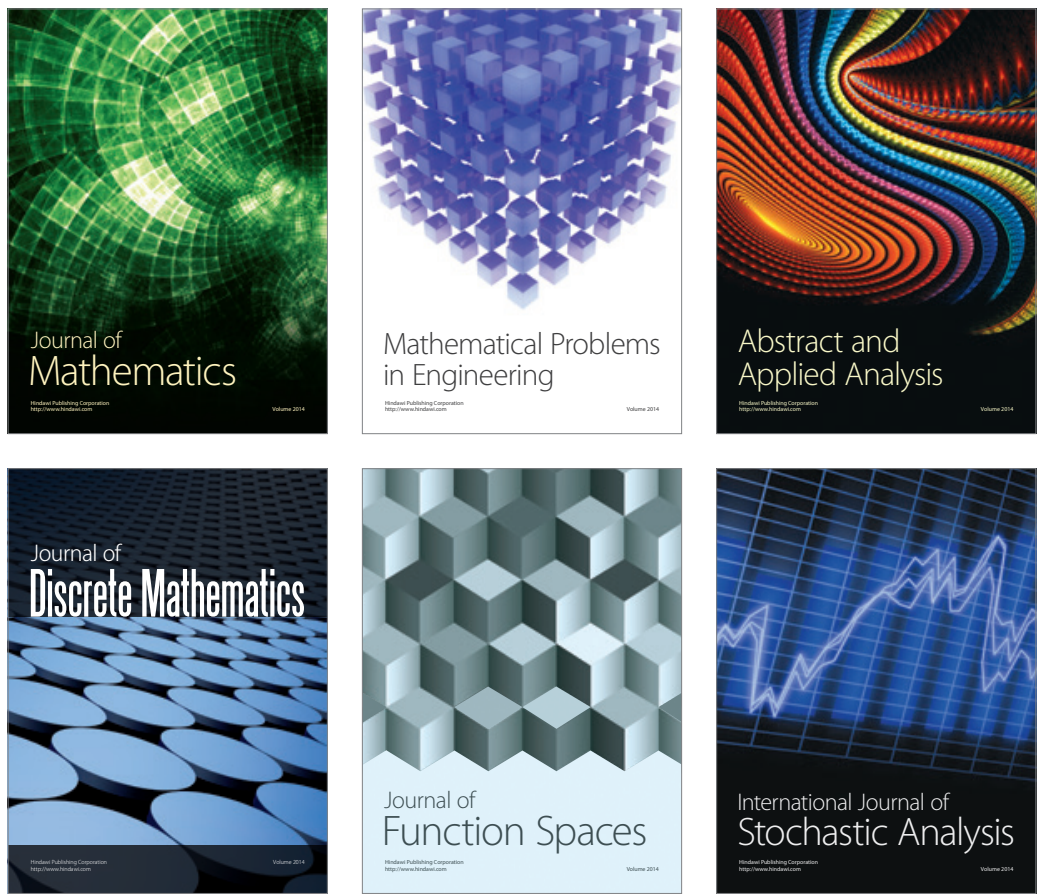

Journal of

Function Spaces

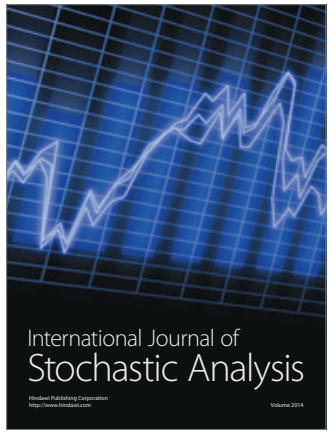

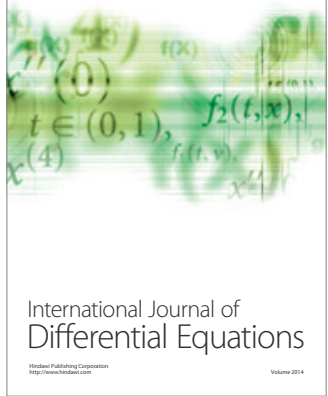
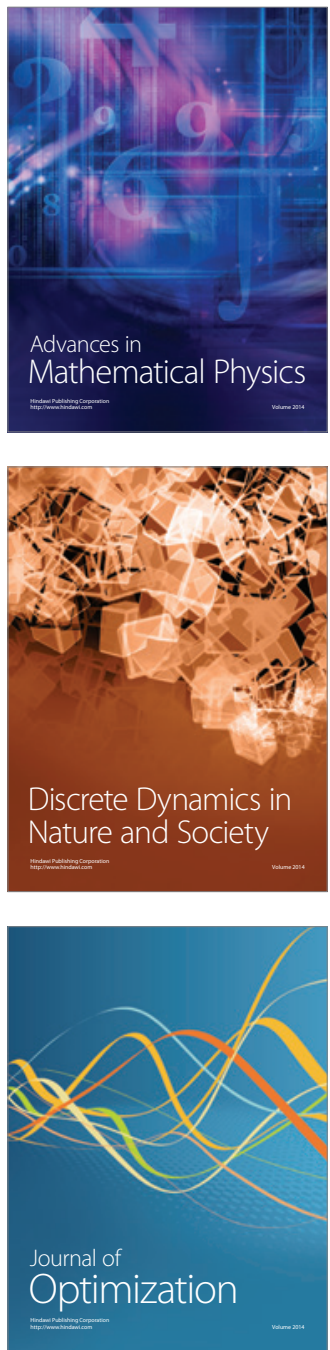